\title{
Three-dimensional printing of navigational template in localization of pulmonary nodule: A pilot study
}

\author{
Lei Zhang, MD, Mu Li, MD, Zeyao Li, MD, Xiermaimaiti Kedeer, MD, Long Wang, MD, Ziwen Fan, MD, \\ and Chang Chen, $\mathrm{PhD}$
}

\section{ABSTRACT}

Background: Small pulmonary nodules are a common problem, especially with the wide implementation of lung cancer-screening program. This poses a great challenge to thoracic surgeons because of the difficulty of nodule localization. We recently built an efficient, customized navigational template using 3-dimensional (3D) printing technology to facilitate the procedure of lung nodule localization. This study aims to investigate its feasibility in clinical application.

Methods: Patients with peripheral lung nodules $(<2 \mathrm{~cm})$ were enrolled. Preadmission computed tomography images were downloaded and reconstructed into a 3D model. A digital model of the navigational template was designed via computer-aided design software and then exported into 3D printer to produce physical template. The precision of the template-guided nodule localization and associated complications were evaluated.

Results: A total of 16 patients were enrolled, and 18 nodules were localized through template-guided localization. The success rate of lung nodule localization was $100 \%$, and the median time of localization was 13 minutes (range 10-16 minutes). In our series, no significant complication occurred, except for 2 asymptomatic pneumothoraxes. The median deviation between the localizer and the center of the nodule was $10.0 \mathrm{~mm}$, ranging from 5 to $20 \mathrm{~mm}$.

Conclusions: This novel navigational template created by $3 \mathrm{D}$ printing technology is feasible, and it has acceptable accuracy for the application in lung nodule localization. The use of this navigational template could facilitate the procedure of lung nodule localization and may potentially break the dependence of percutaneous localization on computed tomography scanning. (J Thorac Cardiovasc Surg 2017;154:2113-9)

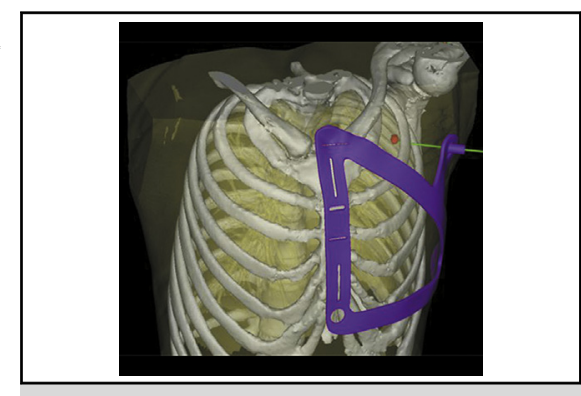

Lung nodule localization via a 3-dimensional printed navigational template.

\section{Central Message}

With 3-dimensional printing technology, a navigational template was designed and printed for small lung nodule localization and showed promising potential for clinical application.

\section{Perspective}

Localization of small lung nodules has become a real challenge to thoracic surgeons since the advancement of computed tomography technology used for lung cancer screening program. A 3-dimenstional printed navigational template in lung nodule localization could significantly facilitate the procedure of percutaneous lung nodule localization and may have the potential to alter the field of nodule localization.

See Editorial Commentary page 2120
With the widespread availability of lung cancer-screening programs, small lung nodules have become much more prevalent in clinical scenarios. It is difficult to localize these

\footnotetext{
From the Department of Thoracic Surgery, Shanghai Pulmonary Hospital, Tongji University School of Medicine, Shanghai, People's Republic of China. Supported by National Natural Science Foundation of China (NSFC 81570014). Drs Zhang and Li equally contributed to this work.

Received for publication Jan 21, 2017; revisions received Aug 5, 2017; accepted for publication Aug 19, 2017; available ahead of print Oct 7, 2017.

Address for reprints: Chang Chen, PhD, Department of Thoracic Surgery, Shanghai Pulmonary Hospital, Tongji University School of Medicine, Shanghai 200443, China (E-mail: chenthoracic@163.com). 0022-5223/\$36.00

Copyright (C) 2017 Published by Elsevier Inc. on behalf of The American Association for Thoracic Surgery

http://dx.doi.org/10.1016/j.jtcvs.2017.08.065
}

nodules merely through palpation during video-assisted thoracoscopic surgery (VATS) because of their small volume and non-solid property. ${ }^{1}$ Therefore, various preoperative localization methods have been developed to localize small lung nodule, allowing for wedge resection by VATS. ${ }^{2-4}$ Among them, hookwire localization guided by computed tomography (CT) scan is currently the most commonly performed technique. ${ }^{5}$ Despite its effectiveness,

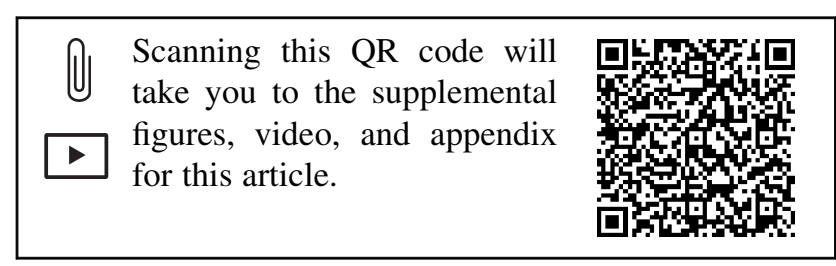




$$
\begin{aligned}
& \text { Abbreviations and Acronyms } \\
& \begin{aligned}
\text { 3D } & =3 \text {-dimensional } \\
\text { BMI } & =\text { body mass index } \\
\text { CT } & =\text { computed tomography } \\
\text { VATS } & =\text { video-assisted thoracoscopic surgery }
\end{aligned}
\end{aligned}
$$

it needs to be performed in CT room, like the other percutaneous localization methods, which to some extent increases the complexity of the procedure, burdens the radiology department, and exposes patients to extra radiation.

Hideo Kodama of Nagoya Municipal Industrial Research Institute $^{6}$ first described 3-dimensional (3D) printing technology in 1981. Since its invention, this technology has been used successfully for preoperative planning, intraoperative guidance, and medical education, showing promising potential in the medical field. ${ }^{7-10}$ The application of 3D printing technology in medical field begins from designing 3D models, which usually are created by converting digitized medical images, such as magnetic resonance imaging or CT images, into computer-aided design software. Thus, it gives $3 \mathrm{D}$ printing the advantages of customizability and the ability to produce complex models.

With this knowledge in mind, we conceived using 3D printing technology to design a navigational template to mark the puncture site and angle of the localizer before lung nodule localization. If it were practical, we would be able to facilitate the process of lung nodule localization and may even localize the lung nodule outside the CT room. To test this idea, we designed and printed a novel navigational template for peripheral lung nodule localization using $3 \mathrm{D}$ printing technology. This article aims to demonstrate this novel template-guided nodule localization method and examine its feasibility in clinical application.

\section{PATIENTS AND METHODS \\ Template Design and Printing}

The design of 3D template model begins with the procurement of the patients' thoracic CT images. After obtaining patient's consent to participate in the study, we downloaded patient's CT images from Picture Archiving and Communication Systems. The CT scan with slice thickness of $1.5 \mathrm{~mm}$ was taken routinely for patients with small lung nodules in our hospital. We then used Bio3D software (Black Ship Technology, Co, Ltd; Shanghai, China) to process the obtained CT data and eventually reconstructed patient's thoracic 3D digital model, which was exported as stereolithography file. To process the patients' CT data, we integrated the spatial information of the target lung nodule into the digital model. Based on this thoracic 3D model, we developed the digital model of the navigational template.

Subsequently, we used computer-aided design software to mark the anatomical landmarks on the template to ensure its correct placement of this template on patient's thoracic wall (Figure 1). Detailed steps about the design of this customized template are described in Figures E1 to E6. After we finished the design of this 3D template model, the model was then printed by FS251P printer (Farsoon, Changsha City, China) with nylon (PA3200). The cost of creating 3D template per patient ranged from 80 to 100 US dollars. It usually took about 1 hour to develop the digital model of the customized template and 4 to 6 hours to print the physical copy depending on the size of the template. The physical copy of this template is shown in Figure 2, A.

\section{Study Population}

Both the radiologist and the referring surgeon assessed the eligibility of the patients with peripheral lung nodule who required diagnostic pulmonary wedge resection by VATS between July 1, 2016, and September 30, 2016. The inclusion criteria were as follows: (1) the maximum diameter of the target lung nodule was less than $20 \mathrm{~mm}$; (2) the minimum distance from the outer edge of the nodule to the nearest pleural surface was greater than $10 \mathrm{~mm}$ if the target nodule was solid but not subsolid (mixed nodule or pure ground glass opacity); (3) the target nodule was not located in the regions of the scapula where the bone structure impeded the percutaneous localizer; and (4) the inner edge of the target nodule was at least $2 \mathrm{~cm}$ away from major pulmonary arteries and veins to allow secure lesion excision.

If the inclusion criteria were met, a trained research assistant approached the patient, as a potential study participant, at least 2 days before the scheduled surgery. The patient was then informed about the risks and benefits of the procedure, and written informed consent was obtained. Once the patient consented to participate in the study, we started the process of template design and production, which took about 1 day to complete. The Institutional Review Board (IRB No. K16-302) at Shanghai pulmonary hospital approved the study.

\section{Localization Procedure}

An interventional radiologist and surgeon performed preoperative localization in radiology department on the day of the surgery. After positioning the patient, the navigational template was placed on the patient's thoracic wall (Figure 2, B). During this step, the practitioner needed to carefully identify patient's anatomical landmarks to guarantee the correct placement of the navigational template. In the next step, we immobilized the navigational template to patient's thoracic wall by using medical adhesive tape in case of incidental deviation.

Subsequently, an initial CT scan was performed and the practitioner pre-evaluated the precision of template-guided localization by drawing an imaginary line from the puncture point toward the lung. Subsequently, the deviation between the imaginary line and the center of the target nodule was measured (Figure 3,A). Based on our previous experience of CT-guided localization, deviation of less than $2 \mathrm{~cm}$ was considered precise enough to allow for safe nodule localization. Afterward, we administered local anesthesia and inserted the hookwire after patient's deep inspiration. However, if deviation of more than $2 \mathrm{~cm}$ was found on the initial CT scan, it was recorded as the failure of the template-guided method; hence, routine CT-guided hookwire localization was used to localize the target lung nodule.

After the completion of nodule localization, a second CT scan was performed to confirm the correct insertion of the localizing needle, check for pneumothorax and hemorrhage, and evaluate the precision of nodule localization by measuring the deviation from the localizer to the center of the target lung nodule (Figure 3, B). The length of procedural time was measured from the time a patient was lying on the examining bed of the CT scanner to the time he/she received the second CT scan to confirm the localizer placement. Detailed information about the procedure of template-guided nodule localization was presented in both Appendix E1 and Video 1.

\section{Statistical Analysis}

Continuous data were expressed as the median with interquartile ranges and analyzed with the Mann-Whitney $U$ test. Categorical variables were analyzed with the Fischer exact test. All statistical analyses were 2-tailed 

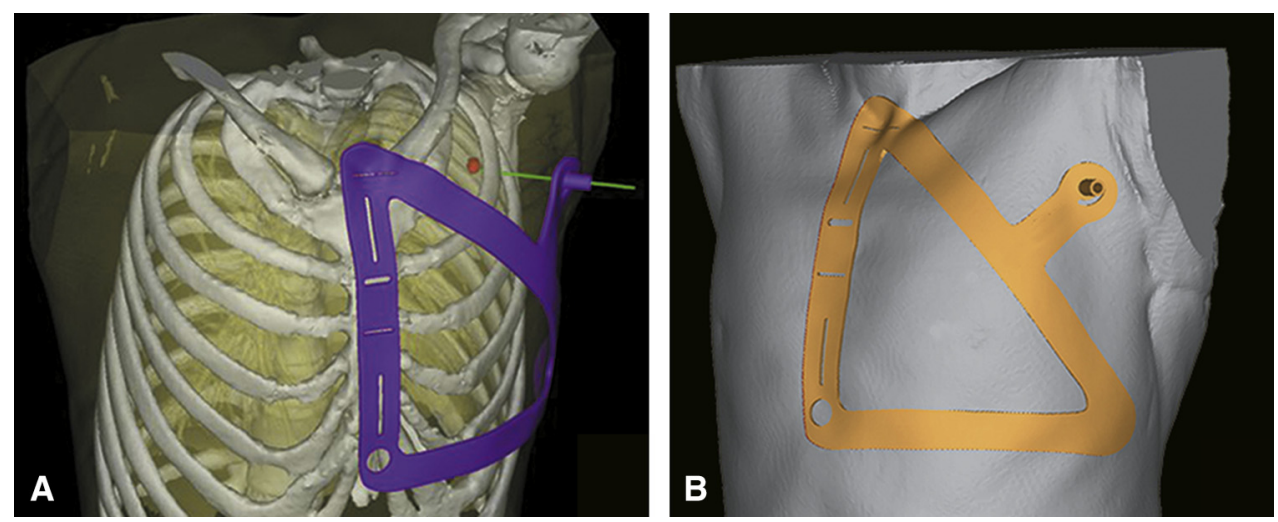

FIGURE 1. The scheme of template design. A, Reconstruction of the thoracic structure (the red dot signified as the target nodule). B, Digital model of the navigational template.

with a significant level of 0.05 , and they were conducted via SPSS statistical package software (version 19.0; IBM SPSS Inc, Armonk, NY).

\section{RESULTS}

Between July 2016 and September 2016, 26 patients with indeterminate lung nodule were approached, and 22 patients provided consent to participate. Six patients dropped out of the study due to being unfit for surgery $(\mathrm{n}=1)$, missing template $(\mathrm{n}=2)$, or unplanned changing of surgical schedule $(\mathrm{n}=3)$. Eventually, 16 patients underwent preoperative nodule localization by navigational template-guided localization with subsequent lung wedge resection. Eighteen lung nodules were actually localized under the guidance of navigational template because 2 patients had 2 separate nodules. Patients' baseline and nodule characteristics are summarized in Table 1.

The sex distribution of the study population was almost equal, with $44 \%$ male patients. The median age was
57 years, and the median body mass index (BMI) value was 22.7, ranging from 18.0 to 26.3. Most nodules were pure ground glass opacity $(12 / 18)$, and the most common location was right upper lobe (8/18). The median diameter of nodules was $11.0 \mathrm{~mm}$ (range $6-16 \mathrm{~mm}$ ), and the median distance from the outer edge of nodule to pleural surface was $11.5 \mathrm{~mm}$ (range $0-29 \mathrm{~mm}$ ).

All nodules were localized successfully under the guidance of navigational template and the median length of localization procedure was 13 minutes (range 10-16 minutes). The median deviation between the localizer and the center of the lesion was $10.0 \mathrm{~mm}$, ranging from $5.0 \mathrm{~mm}$ to $20.0 \mathrm{~mm}$. The correlation between BMI value and deviation was nonsignificant (Spearman $\mathrm{r}=0.07, P=.82$ ). For the nodules in the upper/middle lobe and lower lobe, the median deviation was 9.0 and $15.0 \mathrm{~mm}$, respectively (Table 2). However, the difference in deviation between these 2 groups was nonsignificant $(P=.49)$. Regarding the complication
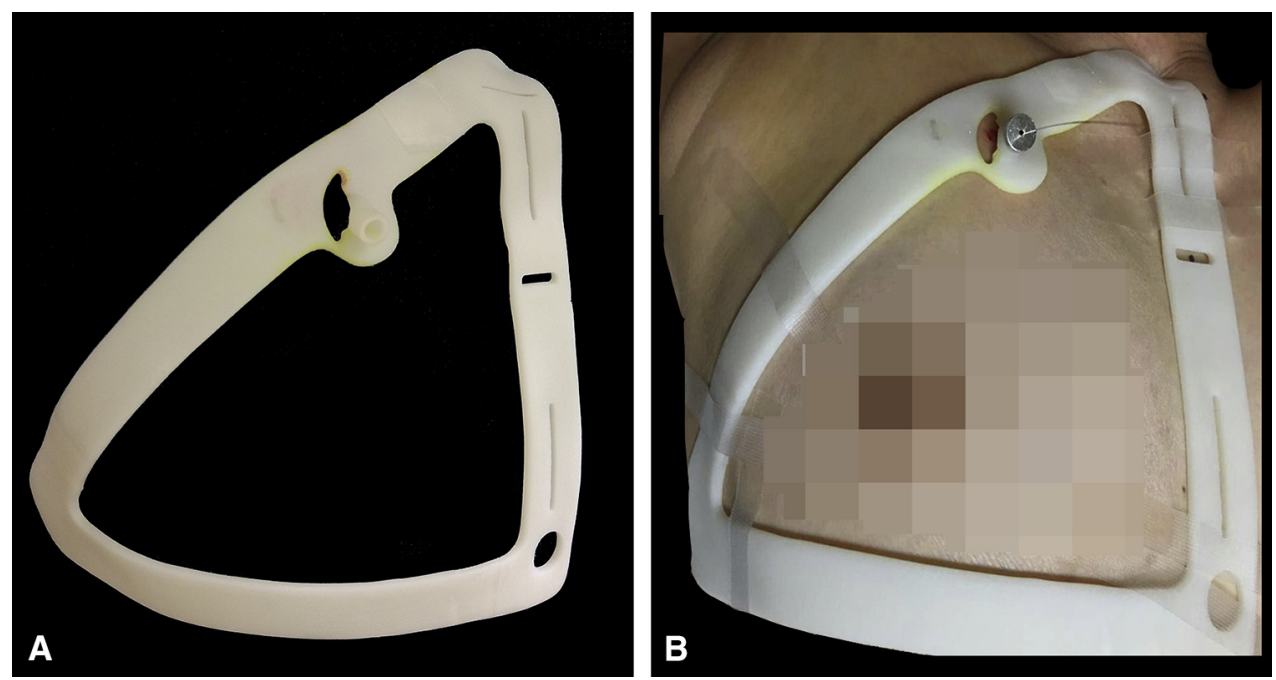

FIGURE 2. The process of lung nodule localization using navigational template. A, Navigational template. B, Needle insertion under the guidance of template. 

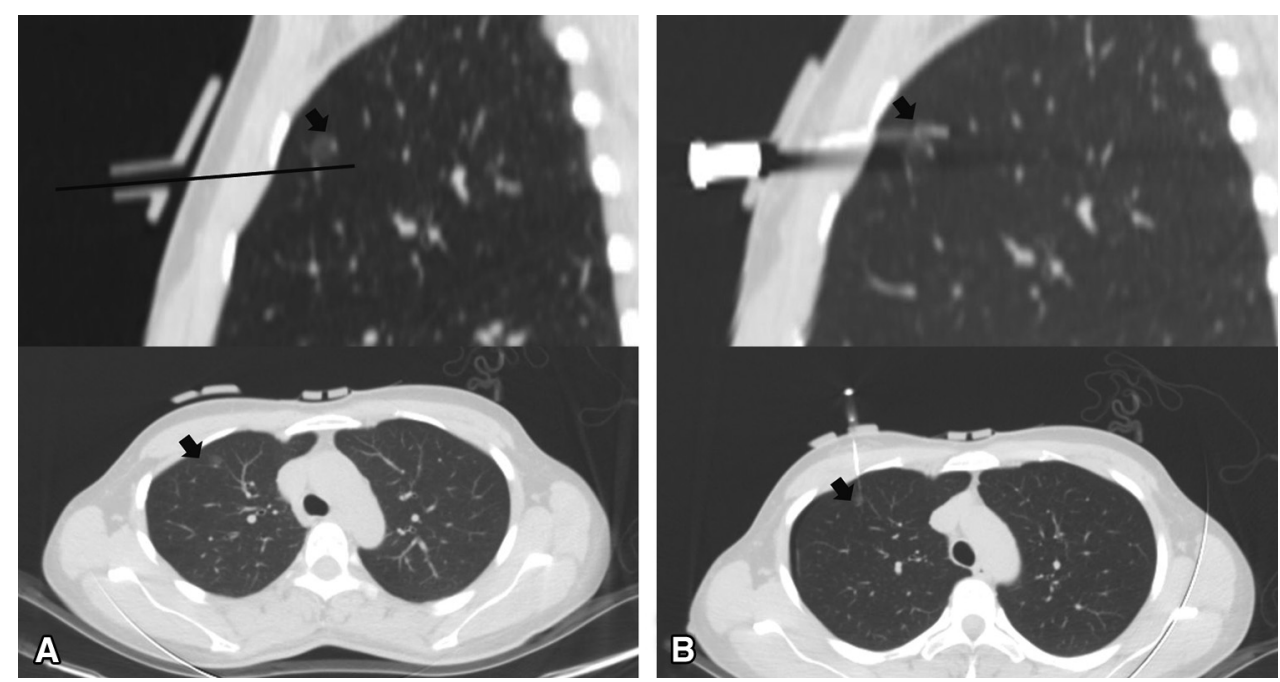

FIGURE 3. The precision of template-guided localization evaluated by CT scanning. A, CT scan before needle insertion. B, CT scan after needle insertion. (Black arrow indicates the target nodule.)

associated with lung nodule localization, 2 patients had asymptomatic pneumothorax on the CT scan, which did not need further intervention. No other complications occurred during template-guided localization.

After nodule localization, patients were wheeled into the operating room, where they underwent lung wedge resection by VATS. The median waiting time between localizer insertion and operation was 120 minutes (range 60-240 minutes). No hookwire dislodgement was observed, and all patients successfully underwent wedge resection under the guidance of hookwire. Intraoperative frozen section analysis revealed that, of the 18 lung nodules, 2 nodules were diagnosed as atypical adenomatous hyperplasia, 7 as adenocarcinoma in situ, 2 as minimally invasive adenocarcinoma, 3 as invasive adenocarcinoma, and 4 nodules were benign. We further performed lobectomy and mediastinal lymph dissection in 3 patients with adenocarcinoma. Concerning patients with

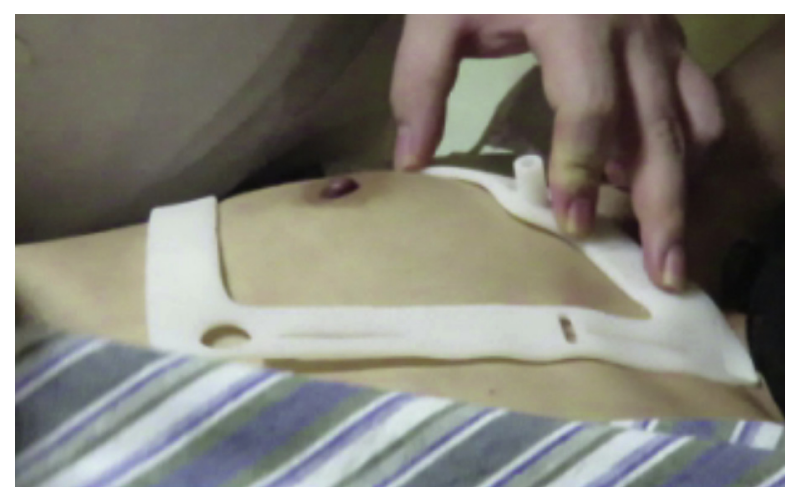

VIDEO 1. Lung nodule localization using 3-dimensional printed template. Video available at: http://www.jtcvsonline.org/article/S00225223(17)31827-5/fulltext. adenocarcinoma in situ or minimally invasive adenocarcinoma, we routinely performed lung wedge resection and lymph node sampling as the curative surgical approach in our center. Among the 12 oncologically related nodules (excluding the 4 benign nodules and 2 adenomatous hyperplasia), frozen section analysis revealed both the nodule size and margin distance. The median diameter of these 12 nodules was $7.0 \mathrm{~mm}$, ranging from 5.0 to $12.0 \mathrm{~mm}$, and the median margin distance was 17.5 , ranging from 12.0 to $25.0 \mathrm{~mm}$ (Table 3). The margin distance was larger than the diameter of the nodule in all resected specimen.

TABLE 1. Clinical and radiologic characteristics of the population

\begin{tabular}{lc}
\hline \multicolumn{1}{c}{ Variables } & n \\
\hline Sex, \% male & $44 \%(7 / 16)$ \\
Median age, y & $58(49-62)$ \\
\hline BMI & $22.7(19.5-25.0)$ \\
Nodule size, mm & $11.0(8.8-13.5)$ \\
Distance between the outer edge & $11.5(10.0-12.5)$ \\
$\quad$ of nodule and pleura, mm & \\
Nodule morphology & \\
Pure GGO & 12 \\
Mixed nodule & 2 \\
Solid nodule & 4 \\
Location & \\
RUL & 8 \\
RML & 3 \\
RLL & 4 \\
LUL & 1 \\
LLL & 2 \\
\hline
\end{tabular}

Age, BMI, nodule size, and the distance between the outer edge of nodule and pleura were expressed as median (25th-75th percentile). BMI, Body mass index; $G G O$, ground glass nodule; $R U L$, right upper lobe; $R M L$, right middle lobe; $R L L$, right lower lobe; $L U L$, left upper lobe; $L L L$, left lower lobe. 
TABLE 2. Assessment of the precision of lung nodule localization

\begin{tabular}{lcc}
\hline \multicolumn{1}{c}{ Lesion } & n & $\begin{array}{c}\text { Distance between localizer and } \\
\text { the center of the nodule, } \mathbf{~ m m}\end{array}$ \\
\hline All nodules & 18 & $10.0(5.8-16.0)$ \\
RUL + RML + LUL & 12 & $9.0(6.0-11.8)$ \\
RLL + LLL & 6 & $15.0(5.0-19.3)$ \\
\hline
\end{tabular}

The distance between localizer and the center of the nodule was expressed as median (25th - 75th percentile). $R U L$, Right upper lobe; $R M L$, right middle lobe; $L U L$, left upper lobe; $R L L$, right lower lobe; $L L L$, left lower lobe.

\section{DISCUSSION}

With the widespread availability of lung cancerscreening program, small lung nodules have become much more prevalent than ever before in clinical practice, which causes a great concern among patients as well as referring clinicians. Excisional biopsy via VATS generally is performed to determine the pathology of the lesion. However, small lung nodule localization during VATS is challenging, especially for nodules without pleural indentation or discoloration. It is often time-consuming and frustrating to manually palpate lung parenchyma to localize the target lung nodule. Failure to localize the target nodule apparently results in conversion to an unplanned anatomic segmentectomy, lobectomy, or open thoracotomy. Suzuki and colleagues ${ }^{11}$ reported that if the target nodule is smaller than $10 \mathrm{~mm}$ in diameter and the distance to pleura is more than $10 \mathrm{~mm}$, the failure rate of lung nodule localization during VATS is $100 \%$, which is also the most common reason for conversion to thoracotomy.

To solve this problem, several strategies have already been developed to localize lung nodule preoperatively or intraoperatively. ${ }^{2-4}$ Because of its simplicity and efficacy, CT-guided hookwire localization is the most frequently used method. ${ }^{5}$ Several studies ${ }^{2,12,13}$ have reported that CT-guided hookwire lung nodule localization has a success rate of more than $95 \%$ and acceptable morbidity rate in lung nodule localization.

TABLE 3. Surgical approach and postoperative pathology

\begin{tabular}{|c|c|}
\hline Variables & $\mathbf{n}$ \\
\hline \multicolumn{2}{|l|}{ Surgical approach } \\
\hline Wedge resection & 15 \\
\hline Lobectomy & 3 \\
\hline \multicolumn{2}{|l|}{ Pathology } \\
\hline Benign & 4 \\
\hline AAH & 2 \\
\hline AIS & 7 \\
\hline MIA & 2 \\
\hline $\mathrm{AD}$ & 3 \\
\hline Nodule size, $\mathrm{mm} *$ & $7.0(6.0-8.0)$ \\
\hline Margin distance, $\mathrm{mm}$ & $17.5(13.5-20.0)$ \\
\hline
\end{tabular}

However, CT-guided hookwire localization has a few limitations, which are similar to those of percutaneous lung nodule localization. The most obvious one is its dependence on the CT scan, which largely limits its clinical application. To perform lung nodule localization by CT-guided nodule localization, the patients would have to commute between the radiology department and operating room, which complicates the localization procedure and exposes patients to excessive radiation. In addition, pneumothorax or hemothorax caused by lung nodule localization may progress and get severe during the time of waiting for the surgery, especially if the scheduled surgery is unexpectedly delayed after nodule localization has been performed. Although the ideal solution is to preform CT-guided nodule localization in the operating room, the availability of intraoperative CT scanner cannot be achieved in most medical institutions.

Using 3D printing technology, we recently designed and printed a navigational template to guide the percutaneous localization of lung nodule. Through this 3D-printed, customized navigational template, we could significantly simplify and facilitate the localization procedure, since this navigational template marks the puncture site and calculates the depth of insertion for localizer. As long as the safety and accuracy of the template-guided localization can be further confirmed, we may even localize small pulmonary nodule in the operative room just before the surgery, circumventing the requirement of performing the localization procedure in radiology department.

To ensure patient's safety and measure the precision of lung nodule localization, we performed the templateguided localization procedure under the surveillance of the CT scan. After we localized the nodules using the template-guided localization in the CT room, we immediately scanned the patients to measure the deviation between the localizer and the center of the lesion. All patients underwent CT scanning only twice compared with more than 3 times of CT scanning in traditional localization method. This decreased the patients' exposure to radiation. Furthermore, localizer inserted only once without needing to be redirected after the needle insertion, which caused less injury to the patients and alleviated the uncomfortable feeling of the patients during nodule localization.

In our study, 16 patients (with 18 nodules) underwent lung nodule localization through template-guided localization, and all nodules were excised successfully with wedge resection through VATS. Using CT scan, we measured the deviation between the localizer and the center of the lesion, as the center of the lesion was the scheduled target of this navigational template. To our knowledge, this is the first research to measure the deviation between localizer and the nodule to precisely evaluate the outcome of lung nodule localization. Our study showed that the 
median deviation between the localizer and our scheduled target was $10.0 \mathrm{~mm}$ (range, 5.0-20.0 mm) and frozen section analysis revealed that all nodules were resected with oncologically safe margin.

During the long process of refining the navigational template and minimizing the deviation, we found several factors that could affect the accuracy of template-guided nodule localization, the most apparent one being patient's respiratory cycle. The designed template is, in fact, a static copy of the patient's movable thoracic wall at the time of deep inspiration. Because of the shift of diaphragm during patient's respiratory cycle, the location of lung nodule relative to the thoracic wall also shifts, especially for nodules in lower lobe. Therefore, to minimize the deviation resulting from the effect of respiratory cycle, we inserted the localizer after patient's deep inspiration. Despite our efforts to avoid the negative effect of respiratory cycle on deviation in our series, template-guided localization of nodules in lower lobe tended to be less accurate compared to localization of nodules in upper/middle lobe, although the difference in accuracy was insignificant at $P$ value of .49 , which may have been due to the small sample size.

Another factor that could significantly contribute to the deviation between the localizer and the scheduled target is the choice of anatomical landmark, which functions as the reference to correctly place the template on the patient's thoracic wall. Because of the flexibility of patients' skin, we could not place the template merely based on the template fitting the patient's thoracic contour. At the stage of template designing, we reconstructed the bony structure of the patient's thoracic wall and pinpointed the 3D location of the lung nodule relative to the patient's bony structure. Therefore, we placed the template on patient's thoracic wall according to the selected anatomical landmarks even though it may not have fitted precisely the patient's thoracic contour. We found that nodule localization would be precise enough as long as the template matched the anatomical landmarks, regardless of whether the template was completely attached to patient's contour. Because the location of the lung nodule relative to the selected anatomical landmark is constant, we could maximally eliminate the deviation of the template from its predefined position by following this principle. Overall, the deviation, existing in the stage of model design and template placement, has been strategically eliminated or at least minimized.

This study also has several limitations. First, most nodules (except 2) localized through template-guided method located within $20 \mathrm{~mm}$ beneath the pleura. And the deviation would theoretically expand if the insertion route became longer for nodules located deep in the lung. Therefore, the efficacy of template-guided localization for nodules located deep beneath the pleura needs to be confirmed in future study. Second, the BMI value of the enrolled patients was within normal range in this study.
Because anatomical landmarks are sometimes difficult to identify in patients who are obese, this novel application may have limited utility in patients with a high BMI value. Thus, the template-guided localization for obese patient should be explored in future research. Lastly, because of the small number of participants in this study, the precision and safety of this application should be evaluated in a randomized controlled trial in the future. If the localizer placement in template-guided localization can be proven to be as accurate as in CT-guided localization, the ultimate goal of unbinding percutaneous localization from its dependence on CT scan can be achieved.

\section{CONCLUSIONS}

The navigational template built with $3 \mathrm{D}$ printing technology is feasible and has acceptable accuracy in percutaneous lung nodule localization. Through the use of this novel template, we could facilitate the procedure of lung nodule localization and even potentially unbind percutaneous localization from its dependence on CT scan. Randomized clinical trial (ClinicalTrials.gov ID: NCT02952261) to confirm the superiority of templateguided localization under surveillance of CT scan, in terms of localization accuracy, radiation exposure, and procedural length, is underway in patients with solitary lung nodule of less than $2 \mathrm{~cm}$.

\section{Conflicts of Interest Statement}

Authors have nothing to disclose with regard to commercial support.

We thank Shao-Jun Wang from Black Ship Technology, Co, Ltd, for his assistance and technical support in the field of 3D printing technology.

\section{References}

1. Tamura M, Oda M, Fujimori H, Shimizu Y, Matsumoto I, Watanabe G. New indication for preoperative marking of small peripheral pulmonary nodules in thoracoscopic surgery. Interact Cardiovasc Thorac Surg. 2010;11:590-3.

2. Ichinose J, Kohno T, Fujimori S, Harano T, Suzuki S. Efficacy and complications of computed tomography-guided hook wire localization. Ann Thorac Surg. 2013; 96:1203-8.

3. Bolton WD, Howe H III, Stephenson JE. The utility of electromagnetic navigational bronchoscopy as a localization tool for robotic resection of small pulmonary nodules. Ann Thorac Surg. 2014;98:471-5; discussion 475-6.

4. Finley RJ, Mayo JR, Grant K, Clifton JC, English J, Leo J, et al. Preoperative computed tomography-guided microcoil localization of small peripheral pulmonary nodules: a prospective randomized controlled trial. J Thorac Cardiovasc Surg. 2015;149:26-31.

5. Park CH, Han K, Hur J, Lee SM, Lee JW, Hwang SH, et al. Comparative effectiveness and safety of pre-operative lung localization for pulmonary nodules: a systematic review and meta-analysis. Chest. 2017;151:316-28.

6. Kodama H. Automatic method for fabricating a three-dimensional plastic model with photo-hardening polymer. Rev Scientific Instrum. 1981;52:1770-3.

7. Rengier F, Mehndiratta A, von Tengg-Kobligk H, Zechmann CM, Unterhinninghofen R, Kauczor HU, et al. 3D printing based on imaging data: review of medical applications. Int J Comput Assist Radiol Surg. 2010;5: $335-41$. 
8. Kaye R, Goldstein T, Zeltsman D, Grande DA, Smith LP. Three dimensional printing: a review on the utility within medicine and otolaryngology. Int J Pediatr Otorhinolaryngol. 2016;89:145-8.

9. Soon DS, Chae MP, Pilgrim CH, Rozen WM, Spychal RT, Hunter-Smith DJ. 3D haptic modelling for preoperative planning of hepatic resection: a systematic review. Ann Med Surg (Lond). 2016;10:1-7.

10. Wang R, Li G, Liu C, Jia C, Han Y. Three-dimensional printing of reduction template in the contouring of craniofacial fibrous dysplasia. J Craniofac Surg. 2016;27:1792-4.

11. Suzuki K, Nagai K, Yoshida J, Ohmatsu H, Takahashi K, Nishimura M, et al. Video-assisted thoracoscopic surgery for small indeterminate pulmonary nodules: indications for preoperative marking. Chest. 1999;115:563-8.
12. Suzuki K, Shimohira M, Hashizume T, Ozawa Y, Sobue R, Mimura M, et al Usefulness of CT-guided hookwire marking before video-assisted thoracoscopic surgery for small pulmonary lesions. J Med Imaging Radiat Oncol. 2014;58: 657-62.

13. Xu X, Yao Y, Shen Y, Zhang P, Zhou J. Clinical analysis of percutaneous computed tomography-guided hook wire localization of 168 small pulmonary nodules. Ann Thorac Surg. 2015;100:1861-7.

Key Words: three-dimensional printing, localization, lung nodule, navigational template, video-assisted thoracoscopic surgery 


\section{APPENDIX E1.}

In brief, we aim to describe thoroughly the method of designing this navigational template and illustrate the steps of template-guided localization in this study.

First, we describe in detail the workflow of designing this customized template through illustrations for other researchers to repeat. Second, we list the steps of template-guided localization followed by this study, which have been stated in the main body of the manuscript. The reason to cover them again in the Appendix is that we rigorously clarify them with illustrations and emphasize the important aspects that needed to be addressed carefully.

\section{PROTOCOL OF TEMPLATE DESIGN Procurement of Patient's Computed Tomography (CT) Images and Data Importing}

After gaining the patient's consent to participate into the study, we downloaded patient's CT images from Picture Archiving and Communication Systems in the format of Digital Imaging and Communications in Medicine in our hospital. Because the patients with small lung nodules routinely underwent low-dose, high-resolution CT scanning with slice sickness of $1.5 \mathrm{~mm}$, we set CT scan with $1.5-\mathrm{mm}$ cuts as the criterion. No extra CT scan was needed to design the template. Then, we imported the CT images into Bio 3D software and reconstructed 3D models of the thoracic body surface and thoracic bony cage through segmentation function of the software (Figure E1).

\section{Designing the Inserting Route}

The localizing route was designed to "shoot" at the center of the target nodule. Several principles were followed to design the localizing route: (1) to assure that the inserting route within lung parenchyma was the shortest; (2) to avoid injury of obvious pulmonary vessel shown on CT images; and (3) to avoid crossing lobar fissure in case of inflicting extra trauma to the healthy lobe of the lung. The map of designed route also was confirmed by patient's surgeon. Two representative maps demonstrating the inserting route for the nodules in the anterior and posterior of lungs are shown in Figure E2.

\section{Selection of Anatomical Landmarks}

For puncture point in anterior chest, upper edge of the sternum, Louis angle correlated to the second rib, substernal angle, and midline of the sternum were selected as anatomical landmarks to guide accurate placement of the navigational template (Figure E3, Al).

For puncture point in posterior chest, in addition to the anatomical landmarks mentioned previously, posterior midline correlated to spinous process also was selected to ensure accurate placement of the navigational template (Figure E3, A2, B2).

\section{Model Building of the Navigational Template}

Surface extraction/contour extraction. We applied surface extraction according to $3 \mathrm{D}$ model of thoracic body surface built in step 1 (Figure E4, A1, A2, B2).

Thickening of the extracted surface. After obtaining the contour surface of the patient's thoracic body, we then thickened the built surface and the thickness of the template in our study is set as $5 \mathrm{~mm}$ (Figure E4, B1, C2, and D2).

Model of the template. In the final stage, by using a Boolean algorithm, we obtained the template model (Figure E5). The holes or gaps in the template were correlated to the selected anatomical landmarks.

Area of the template for introducing the localizer. To ensure that the inserting angle is exactly as the predesigned, we added a metal introducer sheath (Figure E6), through which the localizer was introduced. The metal introducer sheath was designed such that it is properly accommodated by the protrusion of the template, which defined the inserting angle. By deploying the localizer in this fashion, we could eliminate the possibility that the localizer might unintentionally swing from the designed angle.

Also, the metal introducer can be sterilized with autoclave sterilizer to minimize the risk of infection. There was also an oval-shaped gap in this area of the template, which is designed to facilitate the conducting of local anesthesia (Figure E6).

\section{TEMPLATE-GUIDED NODULE LOCALIZATION Patient Positioning}

Patients with a puncture point in the anterior chest were positioned in supine position on the examining bed of CT scanner, and patients with a puncture point in the posterior chest were positioned in lateral decubitus position. In both situations, the patient's arms were abducted.

\section{Template Placement and Fixation}

After the patient was positioned properly, the template was placed based on the anatomical landmarks described previously. Then, we used medical plaster to attach the template to patient's thorax. (Many fixation methods will work, and the template won't shift easily as long as the patient doesn't move intentionally on the examining bed of CT scanner.)

The template doesn't need to rigorously fit the contour of patient's thorax. And the most important principle about template placement is that the template is placed according to the selected anatomical landmarks. As long as the anatomical landmarks are matched, template-localization will be accurate whether or not the template fits the contour of patient's thorax. (Complete attachment between the template and patient's contour would be difficult because of the inevitability of slight distortion of patient's thorax.) 


\section{First CT Scan to Pre-evaluate the Precision of Localization Before Localizer Deployment}

As soon as the template has been placed correctly and immobilized, the first CT scan is taken to pre-evaluate the precision of localization before the hookwire is inserted. Remember that the $\mathrm{CT}$ scan must be taken with respiration control.

As shown in Figure E7, $A$, and Figure E8, $A$, the hollow cylinder in $\mathrm{CT}$ images (indicated by the white arrow) signifies the puncture point. We drew an imaginary line from the center of this hollow cylinder to the lung and this line indicated the inserting route. If the deviation from this line to the target nodule is less than $20 \mathrm{~mm}$, we then considered that the template-guided localization was precise enough to allow safe nodule resection.

\section{Hookwire Inserting}

After pre-evaluation of template-guided localization, we then inserted the hookwire through the metal introducer sheath (see Figure E6). The most important rule during localizer inserting is that the localizer needs to be inserted after deep inspiration on the part of the patient.

\section{Second CT Scan}

Once the hookwire has been deployed, the second CT scan is taken to confirm localizer placement, rule out significant bleeding or pneumothorax, and measure the deviation between the localizer and the target nodule (See Figure E7, $B$, and Figure E8, $B$ ).

\section{SUPPLEMENTARY FIGURES}

We introduced the workflow of template design by using the CT data of 2 patients (the first patient with puncture point in the anterior chest; the second patient with puncture point in the posterior chest). 

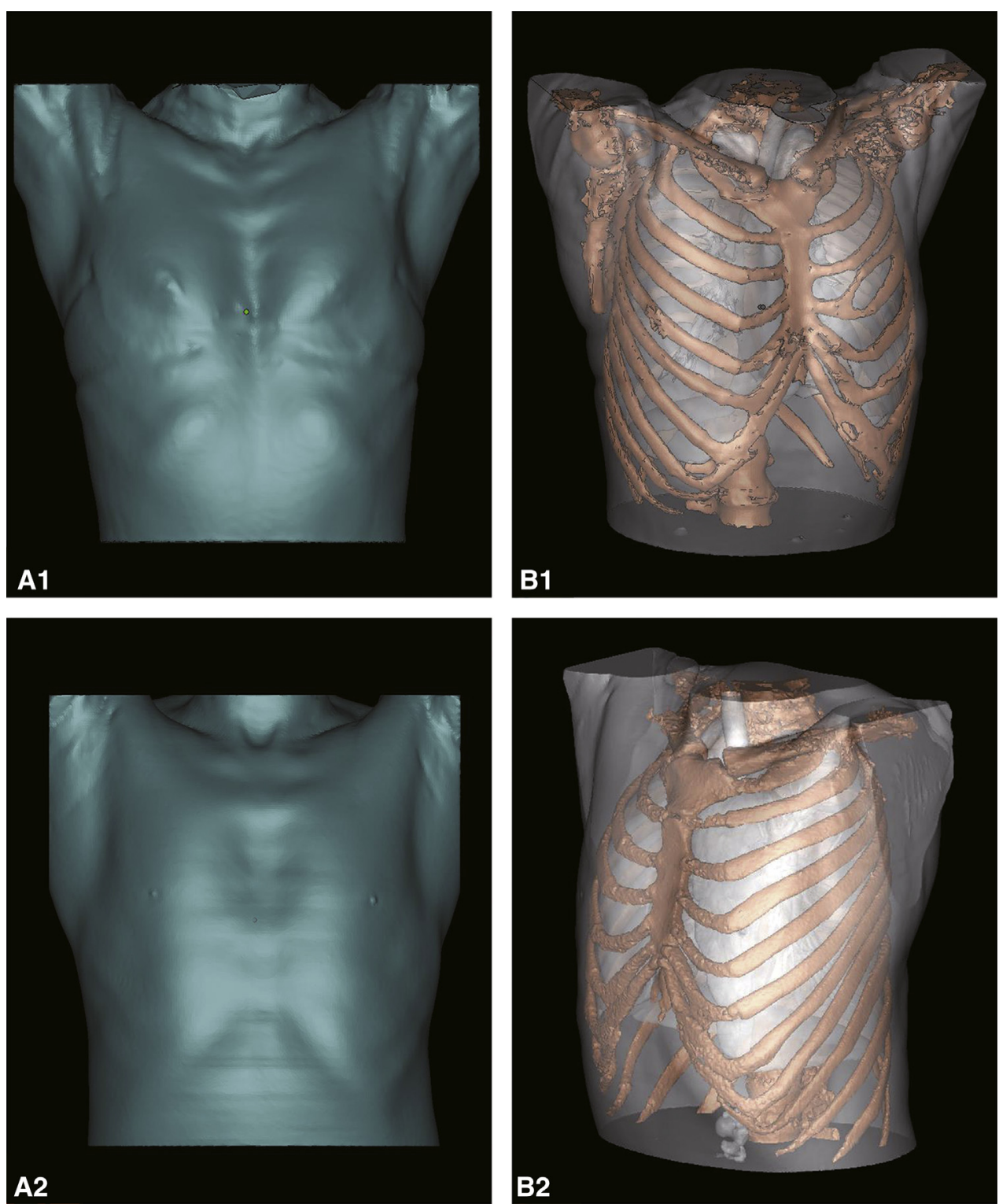

FIGURE E1. 3D model reconstruction of the patient's thorax. A1, 3D model of the thoracic body surface for the first patent. B1, 3D model of thoracic bony cage for the first patient. A2, 3D model of the thoracic body surface for the second patent. B2, 3D model of thoracic bony cage for the second patient. 

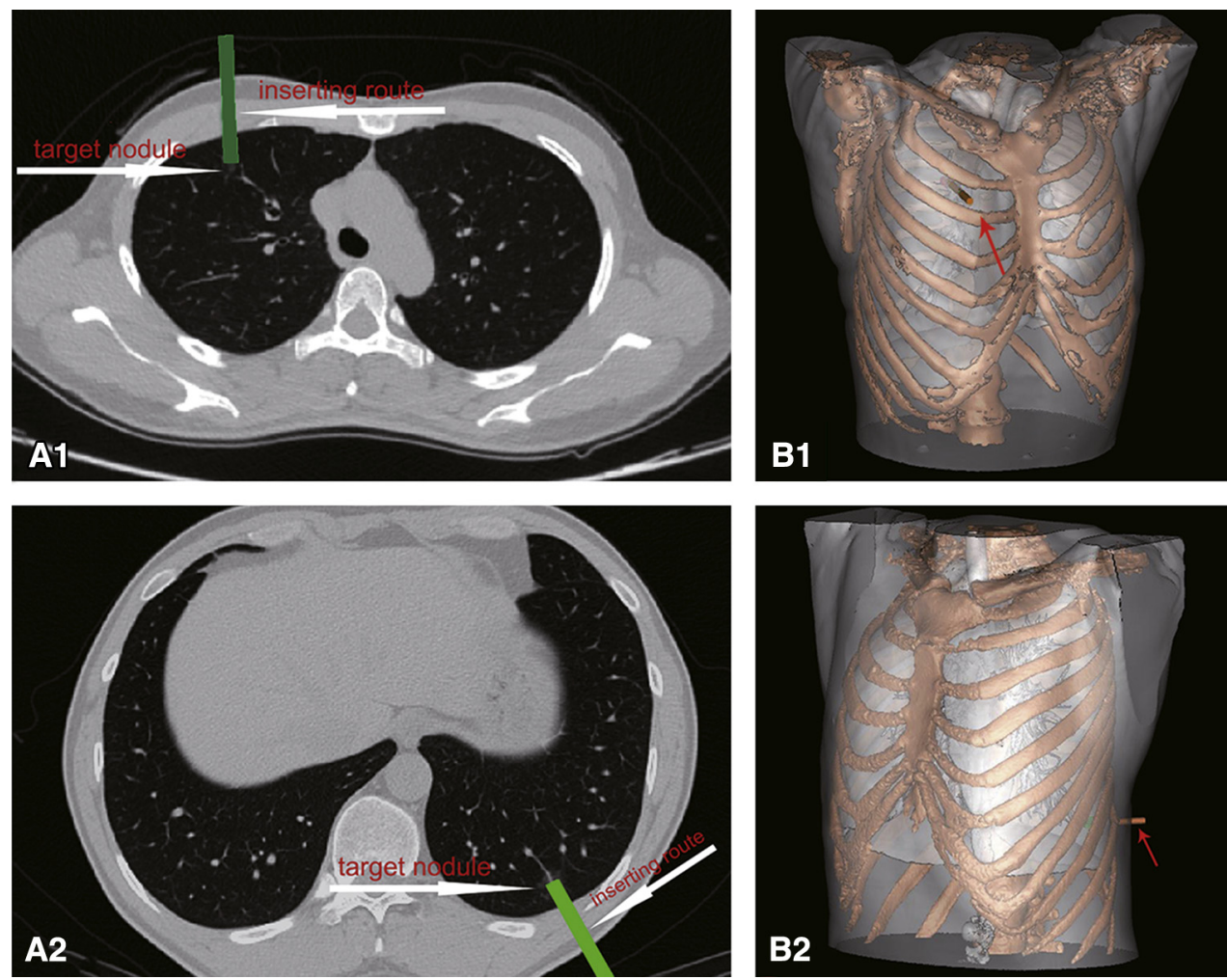

FIGURE E2. Designing of the localizing route. A1, The designed inserting route for the first patient. B1, The designed inserting route shown in the 3D model of the first patient's thorax. B1, The designed inserting route for the second patient. B2, The designed inserting route shown in the 3D model of the second patient's thorax. (Red arrow indicates the inserting route in the reconstructed 3D digital model of the patient's thorax.)
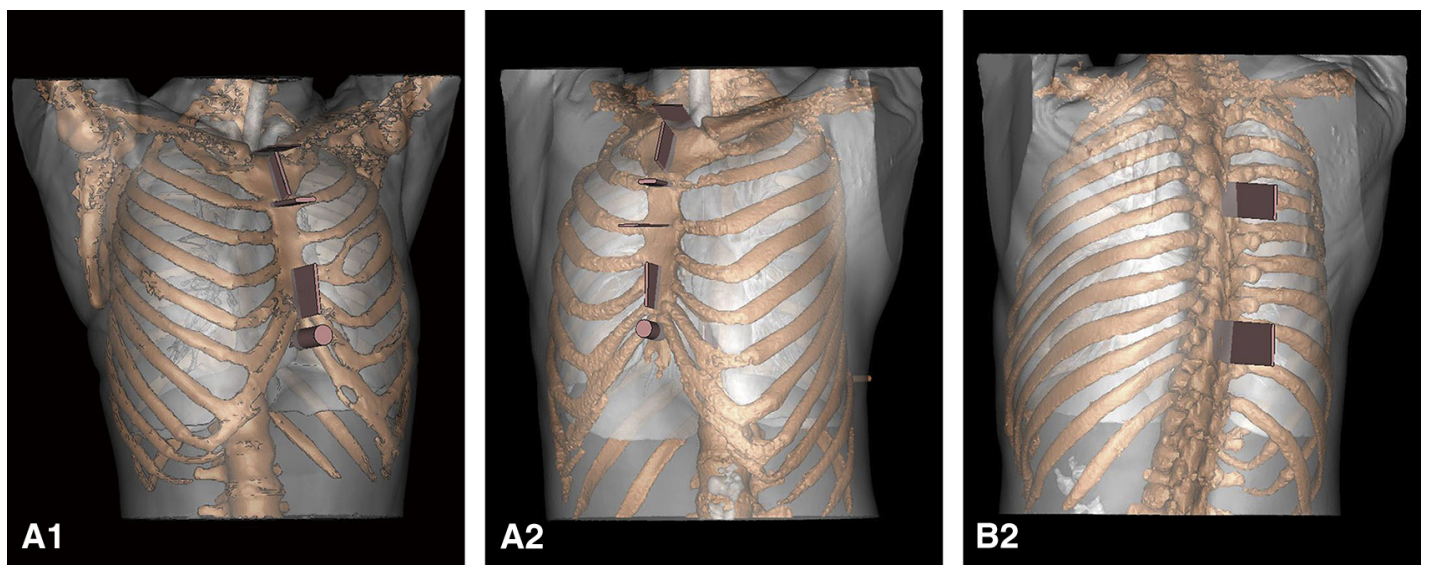

FIGURE E3. The anatomical landmarks selected to guide template placement. A1, The anatomical landmarks for the first patient (upper edge of the sternum, Louis angle, substernal angle, and midline of the sternum). A2, The anatomical landmarks in the anterior chest for the second patient (upper edge of the sternum, Louis angle, substernal angle, and midline of the sternum). B2, The anatomical landmarks in the posterior chest for the second patient (posterior midline). (The extra objects on the 3D model of the patient's thorax demonstrate the identification of the anatomical landmarks during template design.) 

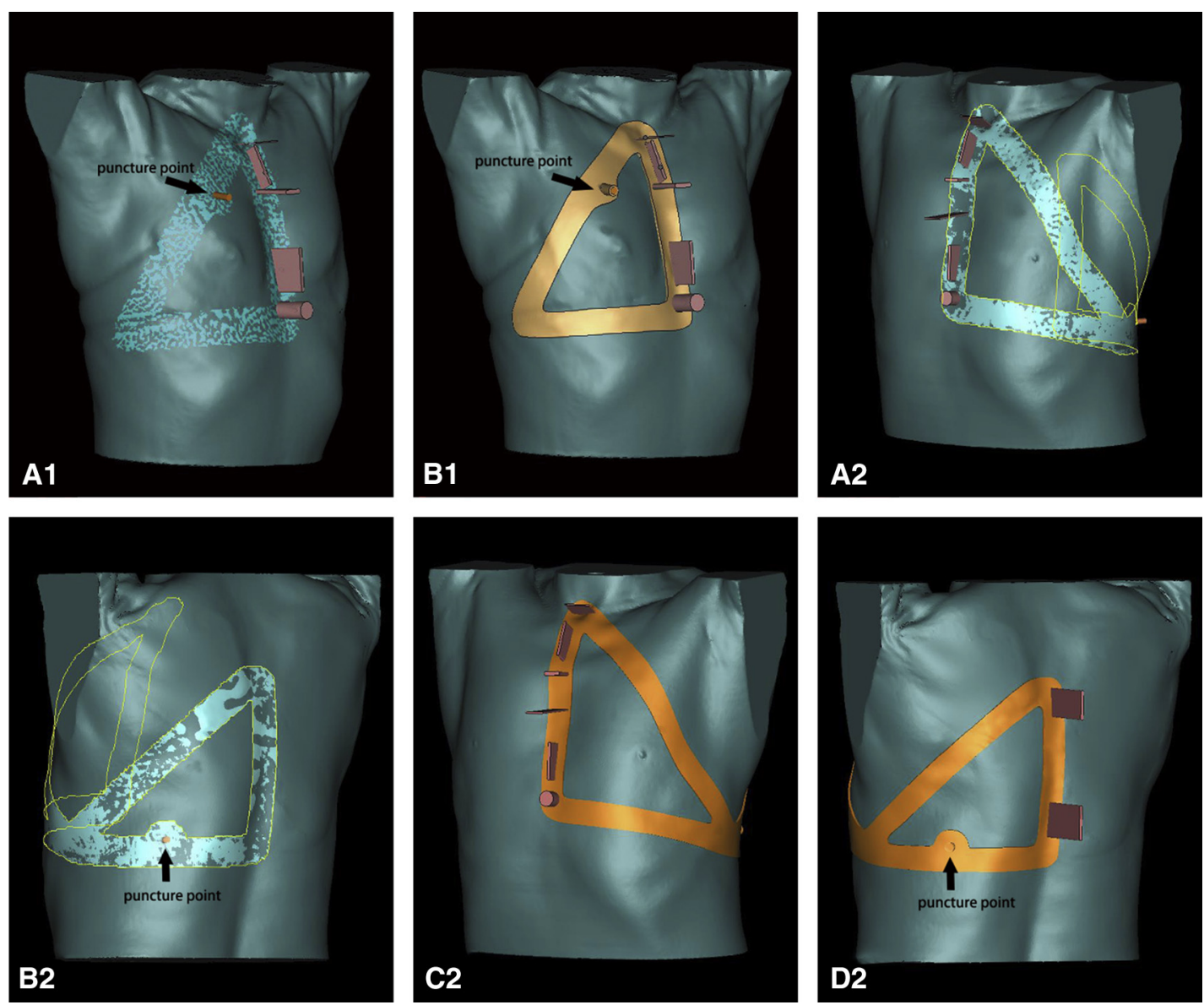

FIGURE E4. Model building of the navigational template. A1, Surface extraction for the first patient. B1, Thickening of the extracted surface for the first patient. A2 and B2, Surface extraction for the second patient. C2 and D2, Thickening of the extracted surface for the second patient.
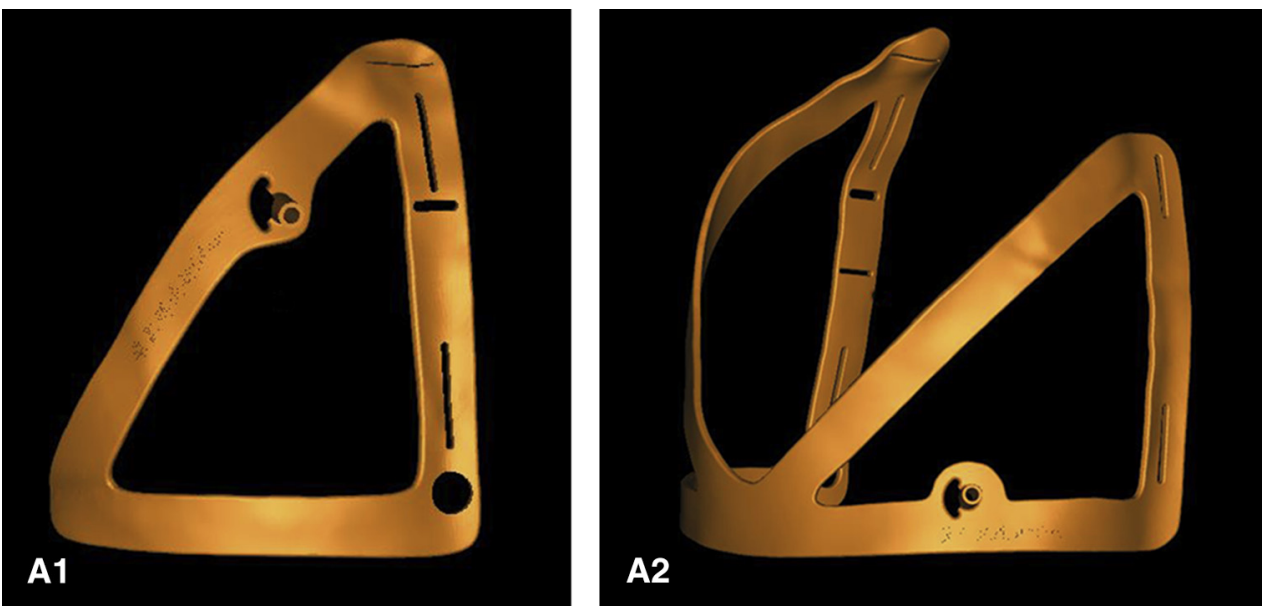

FIGURE E5. Digital model of the navigational template. A1, Digital model of the navigational template for the first patient. A2, Digital model of the navigational template for the second patient. 


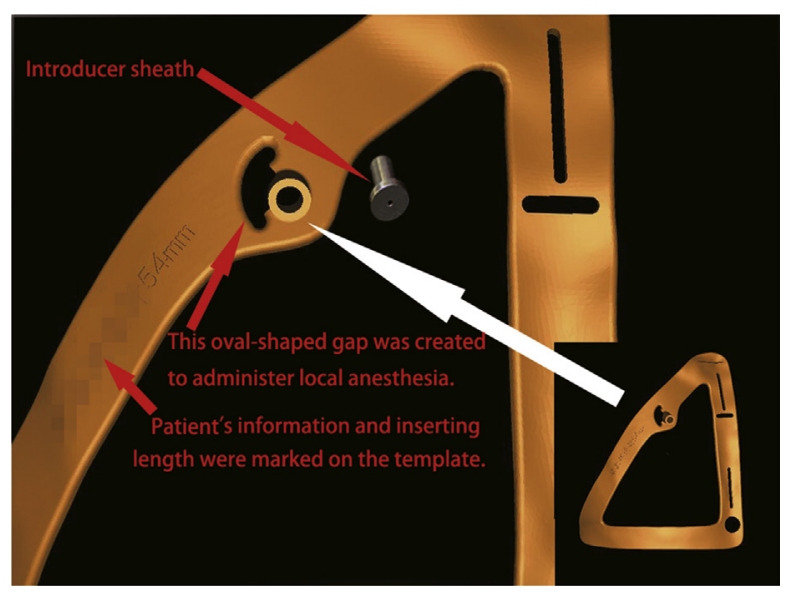

FIGURE E6. Area of the template for introducing localizer. A metal introducer sheath was designed to prevent the localizer from deviating from the predefined angle. An oval-shaped gap also was designed in this area to administer local anesthesia. The patient's information and inserting length for nodule localization were marked on the template to facilitate the localizing procedure.
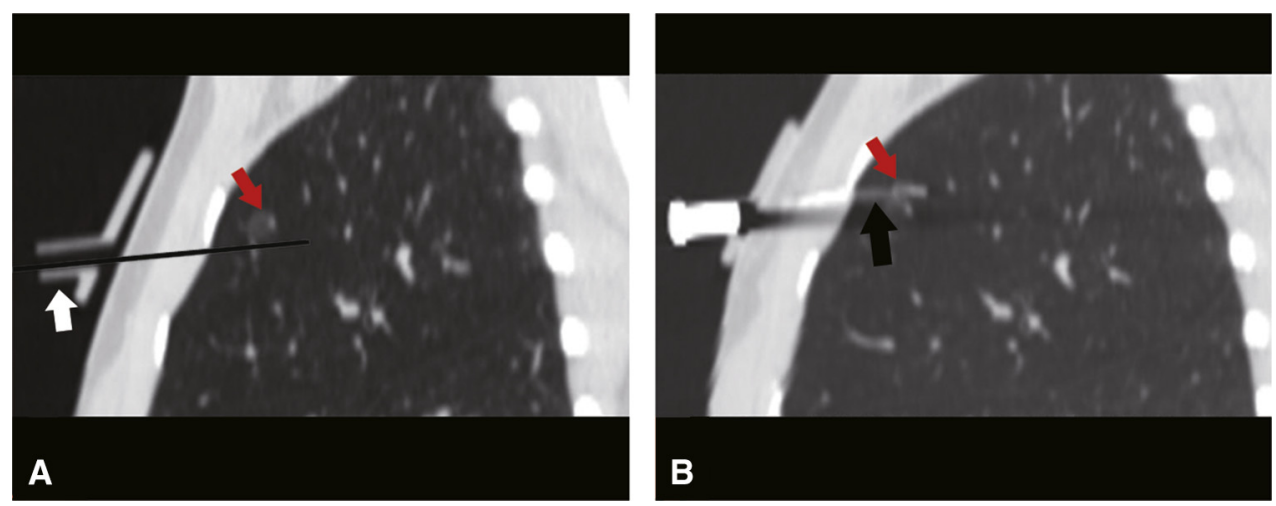

FIGURE E7. Template-guided localization for patient with the puncture point in anterior chest. A, First CT scan to pre-evaluate the precision of nodule localization after template placement and before localizer inserting. B, Second CT scan to confirm the nodule localization after localizer inserting. (Red arrow indicates the target nodule, and black arrow indicates the localizer.). 

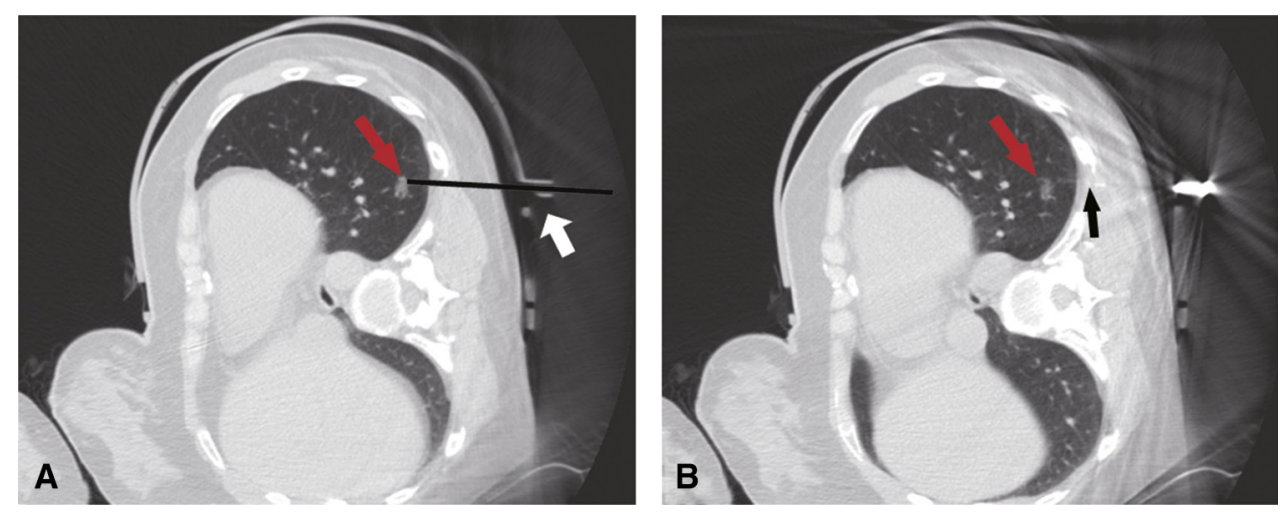

FIGURE E8. Template-guided lung nodule localization for patient with the puncture point in posterior chest. A, First CT scan to pre-evaluate the precision of nodule localization after template placement and before localizer inserting. B, Second CT scan to confirm the nodule localization after localizer inserting. (Red arrow indicates the target nodule, white arrow indicates the area of the template for introducing the localizer, and black arrow indicates the localizer.) 